aims to characterize epidemiology of herpes simplex virus type 2 (HSV-2) in Latin America and the Caribbean.

Methods HSV-2 reports were systematically reviewed and synthesized, and findings were reported following PRISMA guidelines. Meta-analyses and meta-regressions were conducted.

Results 102 relevant reports were identified including 13 overall incidence measures, 163 overall (and 402 stratified) seroprevalence measures, and 7 and 10 proportions of virus detection in genital ulcer disease (GUD) and in genital herpes, respectively. Pooled mean seroprevalence was $20.6 \%$ (95\% confidence interval (CI): 18.7-22.5\%) in general populations, $33.3 \%$ (95\% CI: 26.0-41.0\%) in intermediate-risk populations, $74.8 \%$ (95\% CI: $70.6-78.8 \%)$ in female sex workers, and 54.6\% (95\% CI: 47.4-61.7\%) in male sex workers, men who have sex with men, and transgender people. In general populations, seroprevalence increased from $9.6 \%$ (95\% CI: $7.1-12.4 \%)$ in those aged $<20$ years, to $17.9 \%(95 \% \mathrm{CI}: 13.6-22.5 \%)$ in those $20-30,27.6 \%$ (95\% CI: $21.4-34.2 \%)$ in those $30-40$, and $38.4 \%(95 \%$ CI: $32.8-44.2 \%)$ in those $>40$. Compared to women, men had lower seroprevalence with an adjusted relative risk (ARR) of $0.68 \quad(95 \%$ CI: 0.60-0.76). Seroprevalence declined by $2 \%$ per year over the last three decades [ARR of 0.98 (95\% CI: 0.97-0.99)]. Pooled mean proportions of HSV-2 detection in GUD and genital herpes were $41.4 \%$ (95\% CI: 18.9-67.0\%) and 91.1\% (95\% CI: 82.7-97.2\%), respectively.

Conclusion One in five adults is HSV-2 infected, a higher level than other world regions, but seroprevalence is declining. Despite this decline, HSV-2 persists as the etiological cause of nearly half of GUD cases and almost all of genital herpes cases.

\section{P074 IN VITRO AND IN VIVO EFFICACY OF LINEZOLID ON TREPONEMA PALLIDUM, THE SYPHILIS AGENT}

${ }^{1} \mathrm{~L}$ Giacani* ${ }^{*},{ }^{1} \mathrm{~A}$ Haynes, ${ }^{2} \mathrm{M}$ Vall Mayans, ${ }^{2} \mathrm{M}$ Ubals Cazorla, ${ }^{3} \mathrm{C}$ Nieto, ${ }^{4,5} \mathrm{C}$ Pérez-Mañá, ${ }^{6,7} \mathrm{~L}$ Quintó, ${ }^{1} \mathrm{E}$ Romeis, ${ }^{2,8} \mathrm{O}$ Mitja. 'University of Washington, aDepartment of Medicine, Division of Allergy and Infectious Diseases, Seattle, USA; ${ }^{2}$ Fight Aids and Infectious Diseases Foundation, Hospital Germans Trias i Pujol, Barcelona, Spain; ${ }^{3}$ Laboratorio Reig Jofre, Barcelona, Spain; ${ }^{4}$ Clinical Pharmacology Unit, Hospital, Universitari Germans Trias i Pujol, Institut de Recerca Germans Trias i Pujol (HUGTiP-IGTP), Badalona, Spain; ${ }^{5}$ Department of Pharmacology, Therapeutics and Toxicology, Universitat Autònoma de Barcelona, Barcelona, Spain; ${ }^{6}$ ISGlobal, Hospital Clinic-Universitat de Barcelona, Barcelona, Spain; ${ }^{7}$ Manhiça Health Research Institute (CISM), Maputo, Mozambique; ${ }^{8}$ Lihir Medical Centre-International SOS, Newcrest Mining, Papua New Guinea

\subsection{6/sextrans-2021-sti.208}

Background Penicillin G (PG), the current standard for syphilis treatment, has important drawbacks that hamper public health efforts to curtail the spread of this infection, particularly in low- and middle-income countries where syphilis is still endemic.

Methods To identify viable alternatives to PG, we tested-both in vitro and in vivo-three marketed antibiotics with adequate pharmacokinetic and pharmacodynamic properties to treat syphilis.

Results We found that linezolid, an oxazolidinone overcoming most of the current drawbacks of PG, showed in vitro bactericidal activity at concentrations of $0.5 \mu \mathrm{g} / \mathrm{mL}$ or higher. When administered orally to experimentally infected rabbits, it induced healing of early lesions at a similar time of PG and effectively treated the infection. The hazard ratio for healing was 3.84 (95\% CI $2.05-7.17 ; \mathrm{p}<0.0001)$ compared to untreated controls.

Conclusions Our findings warrant further research to assess the efficacy of linezolid as an alternative to PG to treat early syphilis in clinical settings.

\section{P077 EVALUATION OF THREE AUTOMATED NONTREPONEMAL RAPID PLASMA REAGIN (RPR) TESTS FOR THE DIAGNOSIS OF SYPHILIS}

${ }^{1} \mathrm{M}$ Shukla*, ${ }^{1} \mathrm{~L}$ Pereira, ${ }^{2} \mathrm{~A}$ Gaynor, ${ }^{1} Y$ Sun, ${ }^{3} \mathrm{D}$ Edwards, ${ }^{4} \mathrm{~T}$ Simmons, ${ }^{5} \mathrm{C}$ Andrews, ${ }^{6} \mathrm{P}$ Park, ${ }^{1}$ E Kersh, ${ }^{1} Y$ Fakile. ${ }^{1}$ Centers for Disease Control and Prevention, Atlanta, USA; ${ }^{2}$ Association of Public Health Laboratories, Silver Spring, USA; ${ }^{3}$ Oklahoma State Department of Health, Oklahoma City, USA; ${ }^{4}$ Georgia Public Health Laboratory, Decatur, USA; ${ }^{5}$ Alabama Department of Public Health, Mobile, USA; ${ }^{6}$ University of California San Francisco School of Medicine, San Francisco, USA

\subsection{6/sextrans-2021-sti.209}

Background Automated nontreponemal rapid plasma reagin (RPR) tests were recently introduced in the United States and limited performance data is available.

Methods In collaboration with the Association of Public Health Laboratories, three public health laboratories (PHL) were selected through competitive bidding to evaluate the performance of three FDA-cleared automated RPR tests: AIX-1000 (Gold Standard Diagnostics); ASI Evolution (Arlington Scientific); and BioPlex 2200 Syphilis Total \& RPR assay (Bio-Rad Laboratories) for syphilis testing. CDC prepared three panels that included: a qualitative panel comprised of 455 syphilis reactive/nonreactive specimens; a quantitative panel comprised of 50 syphilis reactive (titer 1:64 to $1: 1024)$ specimens; and a reproducibility panel comprised of 15 nonreactive and reactive (titer $1: 1$ to $1: 64$ ) specimens. Specimens were tested following manufacturers' instructions. For the reproducibility panel, each specimen was tested 10 times. Prior test results were blinded throughout testing to all sites. Results from the automated RPR tests were compared to CDC data obtained by manual RPR (Arlington Scientific) and T. pallidum particle agglutination assays (TPPA, Fujirebio) for analysis.

Results Testing of the qualitative panel demonstrated sensitivity and specificity of $97.5 \%$ and $99.5 \%$ for AIX1000, 91.1\% and 98.5\% for ASI Evolution, and 90.1\% and 99.0\% for Bioplex, respectively, when compared to the manual RPR and confirmed by TPPA. Testing of the quantitative panel showed within range results ( \pm 2 -fold to manual RPR titer) for 94\%, $48 \%, 68 \%$ of specimens by AIX-1000, ASI Evolution and BioPlex, respectively. For the three automated RPR tests, reproducibility testing demonstrated 30-100\% agreement, with Bioplex showing slightly elevated titer when compared to manual RPR.

Conclusion The introduction of automated RPR assays for syphilis could help expediting syphilis testing, reducing workload and interpretation related errors. However, further testing is needed to establish reproducibility and full titer range validation relative to manual RPR. 